\title{
Aplicación del proceso de jerarquía analítica (AHP) para la toma de decisión con juicios de expertos
}

\author{
Application of the Analytical Hierarchy Process (AHP) \\ for decision-making with expert judgment
}

\author{
Adel Mendoza $^{1} \quad$ Cristian Solano $^{1} \quad$ Daniel Palencia $^{1} \quad$ David Garcia $^{1 *}$
}

Recibido 17 de septiembre de 2017, aceptado 18 de julio de 2018

Received: September 17, 2017 Accepted: July 18, 2018

\begin{abstract}
RESUMEN
Cuando se busca una mayor precisión en el análisis de decisiones complejas, se hace necesario recurrir a diferentes técnicas de la Investigación de Operaciones, particularmente en la Teoría de decisiones. Sin embargo, la mayoría de estas pueden ser inadecuadas para manejar desempates de elecciones o disparidad en decisiones grupales por determinadas razones. En este artículo ampliamos los casos de aplicación del esquema metodológico del Proceso de jerarquía analítica (AHP) para abordar problemas en consensos de expertos. Primero, se verifica que esta sea una técnica que garantice el conjunto de preferencias necesarias en un juicio de expertos, luego, aplicamos un procedimiento de priorización usando la media geométrica, para verificar, alcanzar y mejorar el nivel de consenso de grupo por medio de esta técnica de decisión. Finalmente, se ilustra el esquema desarrollado mediante un caso de aplicación en el sector de la logística industrial, llegando a resultados positivos que señalan su efectividad y aplicabilidad dentro del campo de la Teoría de decisiones.
\end{abstract}

Palabras clave: Proceso de jerarquía analítica, teoría de decisiones, investigación de operaciones.

\begin{abstract}
When finely analyzing complex decisions, it is necessary to use several methods of operational research, decision theory in particular. However, these normally cannot handle election tie-breakers or disagreement in group decisions for certain reasons. In this article, we extend the range of the methodological scheme of the analytical hierarchy process (AHP) to address problems in reaching consensus among experts. First, we verify that it is a technique that guarantees the necessary set of preferences in an expert judgment; then we apply a prioritization procedure with the geometric mean to verify, reach and improve the level of consensus of the group via this technique. Finally, through a case of application in the industrial logistics sector, the scheme developed is illustrated, reaching positive results that indicate its effectiveness and applicability within the field of the Decision Theory.
\end{abstract}

Keywords: Analytic hierarchy process, decision theory, operations research.

\footnotetext{
1 Facultad de Ingenierías. Programa de Ingeniería Industrial. Universidad del Atlántico. Km 7 Antigua vía Puerto Colombia. Atlántico, Colombia. E-mail: adelmendoza@mail.uniatlantico.edu.co; cristianjsolano@mail.uniatlantico.edu.co; dapalencia@mail.uniatlantico.edu.co; davidagarcia@mail.uniatlantico.edu.co

* Autor de correspondencia: davidagarcia@mail.uniatlantico.edu.co
} 


\section{INTRODUCCIÓN}

Con el fin de tener estimaciones razonablemente correctas en determinado curso de acción y para reducir la incertidumbre de elecciones erróneas y poco fundamentadas llevadas a cabo dentro de un proceso de toma de decisiones, se hace necesario recurrir a expertos. Un juicio de expertos es, en esencia, un sondeo de opinión entre personas reconocidas como fuentes confiables de un tema, técnica o habilidad, con autoridad en una materia específica [1]. El estudio desarrollado en este manuscrito es una contribución conceptual y metodológica para mejorar y entender procesos complejos de toma de decisiones y su aplicación en juicios de expertos.

Así, esta iniciativa resulta relevante debido a la cantidad de alternativas, opiniones y criterios que se pueden hacer frente a algún objeto de estudio, por lo que se hace necesario seleccionar aquella decisión que mejor se adapte a los objetivos que se pretenden alcanzar, logrando una sinergia entre lo que se quiere hacer y cómo se va a hacer. Sin embargo, ya que lo que se busca es llevar a cabo un proceso de toma de decisiones, estas de manera general, se expresan de manera subjetiva o intersubjetiva, lo cual convierte a un proceso de este tipo en una cuestión de juicio.

Por lo anterior, el objetivo de esta investigación es aplicar un esquema que permita desarrollar un juicio de expertos teniendo en cuenta aquellos elementos que son indispensables en el mismo; haciendo énfasis en el consenso y el desempate de opiniones, y que al mismo tiempo sea sencillo, valido y aplicable para llevar a cabo procesos de decisiones complejos, permitiendo tomar ciertas acciones, bien sea para evitar veredictos sesgados, considerar personal experimentado en ciertos campos, o simplemente reconocer que muchas veces puede que no se cuente con la autoridad o conocimiento necesario para realizar un dictamen. Esto se ve ejemplificado en el caso de estudio presentado posteriormente, en donde al realizar un diseño de un modelo de inventario administrado por el proveedor (Vendor Management Inventory o VMI) para una empresa retail colombiana, fue necesario consultar a un grupo de expertos académicos para la selección de la técnica de modelamiento ideal que sería aplicada para llevar a cabo el proyecto.
Los antecedentes a estudios de este tipo giran en torno a propuestas de aplicación de técnicas de análisis de decisiones a casos muy particulares. Podemos mencionar casos de ejemplo en el sector productivo o dentro de la gestión de la cadena de suministro, en la selección de diversas estrategias [2-6], o hasta implementaciones en la solución a problemáticas sociales relacionadas con la salud, medio ambiente o políticas de responsabilidad social [7-11]. Otros autores han propuesto esquemas de consenso para juicios de expertos, que en su mayoría trabajan en sinergia con el Proceso de análisis jerárquico (AHP), así como la lógica difusa o el método TOPSIS [12-18], o bien con otras metodologías más particulares [19-23]. Del mismo modo, estudios anteriores de este tipo se enfocan en trabajos de investigación de revisión de literatura que giran en torno a estos modelos [24-27].

En consecuencia, se reconoce que muchas de estas técnicas pueden pasar desapercibidas por carecer de practicidad o sencillez en su replicación, al tratarse de enfoques analíticos muy complicados, bien sea en su formulación matemática o ejecución informática. Por su parte, otras presentan cierto tipo de restricciones geográficas o metodológicas, que al ser planteadas, limitan el desarrollo o aplicación de la técnica. También, en algunas la limitante constituye una necesidad de adaptarse a juicios dominantes pese a que ciertos expertos presenten ideas contrarias. Todo ello motiva y sustenta la ejecución de nuevos casos de aplicación que fortalezcan métodos apropiados que se adapten a exigencias específicas.

El presente artículo se divide como sigue: la siguiente sección presenta los conceptos más relevantes relacionados con el juicio de expertos y la selección del Proceso de jerarquía analítica (AHP) como técnica base del esquema propuesto. Consecutivamente, se describen los fundamentos básicos del Proceso de jerarquía analítica, que se complementa con el algoritmo de consistencia y una metodología para sintetizar el juicio de los expertos. De manera posterior, se presenta un caso de aplicación a manera de ejemplo del análisis desarrollado y sus resultados. Finalmente se indican las conclusiones del estudio discutiendo las limitaciones y recomendaciones para investigaciones futuras. 


\section{FUNDAMENTOS DEL JUICIO DE EXPERTOS}

Si bien no hay un acuerdo unánime en relación con la selección de individuos o el mejor método a implementar en alguna situación en particular, primeramente, conviene reconocer que existen diferentes métodos para llevar un juicio de expertos. Entre los más utilizados, según la literatura consultada, está el método de agregados individuales [28-31], en el cual, se le pide a cada experto una estimación directa de su criterio, tratando estadísticamente los datos recogidos. Dicho método no exige una reunión de expertos en un lugar determinado, por lo que no permite su interacción; sin embargo, aporta un gran valor a la hora de evitar sesgos ocasionados por la misma.

Otro de los métodos más populares es el método Delphi [32-35], donde en primera instancia, cada experto responde de manera anónima e individual a cierto cuestionario. Después, se analizan en conjunto sus respuestas, remitiendo la mediana obtenida, así como el intervalo intercuartil y se les pide que reconsideren su juicio anterior teniendo en cuenta estos datos. En cada ronda se repiten tales pasos, y todo juicio individual fuera del intervalo intercuartil tiene que estar justificado. Una débil dispersión de los juicios indica que se ha alcanzado un consenso. No obstante, se observa que la principal desventaja es que puede hacer que los miembros del grupo estén de acuerdo con las personas dominantes, a pesar de tener ideas contrarias cuando reciben una retroalimentación en las distintas iteraciones.

También, se encuentran métodos como el grupo nominal y el consenso grupal [36-38], donde en el primero, se debe reunir a los expertos, los cuales presentarán individualmente sus consideraciones y puntuaciones. Lo siguiente es realizar un debate de los temas tratados, donde cada uno de forma individual y por escrito, puntúa y argumenta su valoración. Finalmente, se procede como con el método Delphi, con la diferencia que permite algún debate entre los expertos. En cuanto al método del consenso grupal, se necesita reunir a un grupo pequeño de expertos en determinado lugar, el objetivo es lograr una puntuación estadística consensuada, permitiendo así, en gran medida, el intercambio de información y opiniones dentro del grupo.

\section{Selección del Método}

Aunque los métodos antes mencionados tienen como fin lograr una valoración consensuada, cada uno tienen los diferentes requerimientos que se exponen en la Tabla 1, entre los cuales los más evidentes son la necesidad de mantener una comunicación constante con los expertos y la necesidad de reunirlos.

Tabla 1. Requerimientos por método.

\begin{tabular}{|l|c|c|}
\hline \multicolumn{1}{|c|}{ Método } & $\begin{array}{c}\text { Comunicación } \\
\text { constante con } \\
\text { los expertos }\end{array}$ & $\begin{array}{c}\text { Reunión de } \\
\text { expertos }\end{array}$ \\
\hline Agregados individuales & NO & NO \\
\hline Delphi & SI & NO \\
\hline Grupo Nominal & SI & SI \\
\hline Consenso Grupal & SI & SI \\
\hline
\end{tabular}

Al considerar necesario una valoración de expertos de diferentes lugares, descartamos los métodos de Grupo Nominal y Consenso Grupal. Similarmente, se descartó el método Delphi, ya que se espera tener una participación amplia de expertos, por lo que una comunicación constante con los expertos en varias rondas resultaría una tarea complicada. Por lo tanto, el único método que cumple con las restricciones para un juicio de expertos apropiado corresponde al método de agregados individuales.

Por otra parte, todo instrumento de medición para un juicio de expertos debe tener tres elementos: un conjunto de alternativas, las cuales serán comparadas por los ellos, también, debe poseer unos criterios cualitativos o cuantitativos, ya que estos serán los puntos de referencias para que cada uno emita sus juicios acerca del análisis en cuestión y, por último, el objetivo del resultado, es decir, el propósito. Además, los roles representan diferentes fuentes de información relevantes para el funcionamiento del debate y selección, el cual es el fin último del método. En adición, los roles poseen cualidades y funciones únicas entre sí, y muy parecidas entre los mismos roles de diferentes juicios, por lo tanto, una adaptación acertada a este esquema de roles en el juicio de expertos corresponde a la propuesta por Rosqvist [39] resumida en la Tabla 2. 
Tabla 2. Roles en el juicio de expertos.

\begin{tabular}{|c|c|}
\hline Rol & Funciones básicas \\
\hline $\begin{array}{l}\text { Tomador de } \\
\text { decisiones }\end{array}$ & $\begin{array}{l}\text { - Presentar el estado del proceso de toma de decisiones y el resultado del objetivo de la decisión grupal. } \\
\text { - Identificar y seleccionar las partes interesadas. } \\
\text { - Definir los recursos necesarios en el proceso. } \\
\text { - Proporcionar los criterios de decisión. }\end{array}$ \\
\hline Juez & $\begin{array}{l}\text { - Seleccionar al grupo de expertos. } \\
\text { - Describir el caso. } \\
\text { - Participa en las discusiones. } \\
\text { - Realizar el informe de decisión. }\end{array}$ \\
\hline Experto & $\begin{array}{l}\text { - Estar familiarizado con el tema. } \\
\text { - Analizar el tema y emitir juicios (de tipo cuantitativo y cualitativo) sobre el mismo. }\end{array}$ \\
\hline
\end{tabular}

\section{PROCESO ANALÍTICO JERÁRQUICO (AHP)}

Debido a la gran cantidad de juicios emitidos por los expertos, se vuelve imperativo resolver un problema de criterios múltiples, con múltiples valoraciones. Por lo anterior, el Proceso analítico jerárquico (Analytic Hierarchy Process o AHP por sus siglas en inglés), es una técnica que se ajusta en gran medida a los requerimientos del problema que se plantea, y que se puede implementar en cinco pasos sistemáticos:

1. Desarrollo de la estructura jerárquica.

2. Representación de los juicios de valor.

3. Construcción de las matrices de juicio de valor y matrices normalizadas.

4. Cálculo de los vectores de prioridad y consistencia.

5. Análisis de resultados.

La estructura jerárquica es una representación gráfica del problema a modo de jerarquía, con el objetivo en la cúspide, las alternativas en el escalón inferior, y los criterios en el nivel (o niveles) intermedios. Lo anterior se presenta en la Figura 1. Por otro lado, debido a la ambigüedad misma de los criterios seleccionados para el juicio de expertos, es imposible priorizar las alternativas de acuerdo con dichos criterios. Dicho esto, se hace necesario utilizar una herramienta que permita los juicios subjetivos, pero al mismo tiempo reduzca la incertidumbre por la naturaleza de los juicios personales.

Por tal motivo, se optó por la escala de Saaty, presentada en la Tabla 3, para definir los valores que pueden tomar los juicios emitidos por los expertos. Esta escala puede considerarse una representación de los juicios de valor para la comparación entre dos alternativas respecto a un criterio dado [40], es decir, para establecer la importancia o preferencia de alternativas en la matriz de comparaciones a pares. Entonces, estas comparaciones pareadas pueden estar basadas en la intuición, datos, análisis previos, o experiencias. De este modo, se puede entregar homogeneidad y cierto grado de certeza a cada una de las comparaciones.

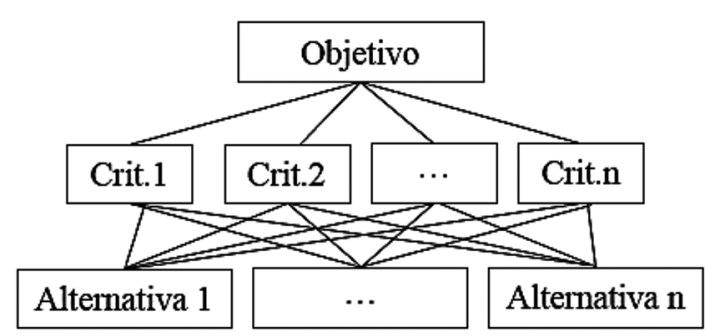

Figura 1. Estructura Jerárquica.

La importancia del AHP está en la determinación de pesos relativos para calificar las alternativas. Al suponer que tenemos $n$ criterios en cierta jerarquía específica, el AHP establece una matriz de comparación por pares A de $n \times n$, que mide el juicio del tomador de decisiones de la importancia concerniente a cada criterio. La comparación por pares se ejecuta de tal modo que el criterio en la fila $i(i=1,2,3, \ldots, n)$ se califica con respecto a cada criterio alterno. Si $a_{i j}$ define el elemento $(i$, $j$ ) de A, el AHP utiliza la escala de Saaty del 1 al 9 , donde $a_{i j}=1$ representa que $i$ y $j$ tienen igual importancia para el experto. Luego, $a_{i j}=5$ indica que $i$ tiene una mayor importancia que $j$ y $a_{i j}=9$ indica que $i$ es extremadamente más importante que $j$. Otros valores intermedios entre estos números 
Tabla 3. Escala de juicio.

\begin{tabular}{|c|l|l|}
\hline Escala numérica & \multicolumn{1}{|c|}{ Escala verbal } & \multicolumn{1}{c|}{ Descripción } \\
\hline 1 & Igual importancia. & $\begin{array}{l}\text { Los dos elementos contribuyen igualmente } \\
\text { a la propiedad o criterio. }\end{array}$ \\
\hline 3 & $\begin{array}{l}\text { El elemento es moderadamente más impor- } \\
\text { tante respecto al otro. }\end{array}$ & $\begin{array}{l}\text { El juicio y la experiencia previa favorecen } \\
\text { a un elemento frente al otro. }\end{array}$ \\
\hline 5 & $\begin{array}{l}\text { El elemento es fuertemente más importante } \\
\text { respecto al otro. }\end{array}$ & $\begin{array}{l}\text { El juicio y la experiencia previa favorecen } \\
\text { fuertemente a un elemento frente al otro. }\end{array}$ \\
\hline 7 & $\begin{array}{l}\text { La importancia del elemento es muy fuerte } \\
\text { respecto al otro. }\end{array}$ & Un elemento domina fuertemente. \\
\hline 9 & $\begin{array}{l}\text { La importancia del elemento es extrema } \\
\text { respecto al otro. }\end{array}$ & $\begin{array}{l}\text { Un elemento domina al otro con el mayor } \\
\text { orden de magnitud posible. }\end{array}$ \\
\hline Incrementos 0,1 & $\begin{array}{l}\text { Valores intermedios entre dos juicios adyacentes. } \\
\text { necesita un alto grado de precisión). }\end{array}$ \\
\hline $\begin{array}{l}\text { Inversos } \\
\frac{1}{2}, \frac{1}{3}, \frac{1}{4}, \frac{1}{5}, \frac{1}{6}, \frac{1}{7}, \frac{1}{8}, \frac{1}{9}\end{array}$ & Se utiliza cuando el segundo elemento es mayor en el criterio a comparar. \\
\hline
\end{tabular}

tienen la notación con la relatividad correspondiente [41]. Además, se debe presentar cierta consistencia en el proceso, si $a_{i j}=k$ entonces $a_{j i}=1 / k$, luego todos los elementos diagonales $a_{i i}$ son iguales a 1 , porque estos califican cada criterio contra sí mismo.

Una vez recopilados los juicios individuales de los expertos, se calculan los pesos relativos de cada criterio, normalizando la matriz para crear una nueva. Este proceso requiere básicamente dividir los elementos individuales de cada columna entre la suma de la columna. Por otro lado, para el cálculo de los vectores de prioridad de cada criterio, es necesario promediar cada fila de la matriz normalizada.

\section{Medida de consistencia}

En adición, es conveniente evaluar la consistencia de la matriz, lo que es un indicador del juicio racional por parte del tomador de decisiones. Para decidir qué nivel de inconsistencia es tolerable o aceptable, debemos considerar una medida de consistencia cuantificable, luego el cálculo del cociente de consistencia para cada matriz se obtiene siguiendo el siguiente algoritmo:

a) Para cada fila de la matriz de comparación, calcular una suma ponderada, con base a la suma del producto de cada elemento por la prioridad calculada de cada criterio.

b) Para cada elemento del vector resultante del paso anterior, dividir su suma ponderada por la prioridad de su criterio correspondiente. c) Determinar la media $\lambda_{\max }$ del resultado del paso anterior.

d) Calcular el índice de consistencia ( $C I$ ) para cada criterio, donde $n$ es igual al número de criterios. La ecuación (1) presenta la fórmula matemática.

$$
C I=\frac{\lambda_{\max }-n}{n-1}
$$

e) Determinar el índice aleatorio (IA), este puede ser calculado de manera empírica como el promedio $C I$ de una muestra grande de matrices de comparación generadas al azar. En la ecuación (2) se denota la expresión.

$$
I A=\frac{1,98(n-2)}{n}
$$

f) Establecer la razón de consistencia $(C R)$ como se presenta en la ecuación (3).

$$
C R=\frac{C I}{I A}
$$

Si el resultado de la ecuación (3) es menor o igual a 0,1 , el nivel de inconsistencia es aceptable, con un resultado contrario se recomienda que el experto debe revisar sus estimaciones. De manera posterior, se deben eliminar aquellas valoraciones de los expertos que presentaron una o más matrices no consistentes, para luego formular las matrices de valoraciones consensuadas, donde el valor de la 
comparación corresponderá a la media geométrica de la comparación de pares de los expertos que no presentaban inconsistencias (como se explica en breve).

\section{Juicio sintetizado y Media geométrica}

Dado que la técnica AHP no permite múltiples valoraciones, se hace necesario una matriz de valoraciones consensuadas. Con varios expertos involucrados, por lo general, no se puede llegar a un consenso y hay que sintetizar o desempatar el juicio. Como ya se ha mencionado, el proceso implica realizar comparaciones entre parejas de alternativas de acuerdo con un criterio dado. Los juicios individuales se hacen comparando una alternativa A, con otra, por ejemplo B, de acuerdo con el criterio dado de cada experto, y el conjunto de todos estos juicios individuales deben ser sintetizados en un solo juicio.

Partiendo de que un experto calcula una relación cuando compara dos componentes, los juicios son ratios. Si los individuos hubieran comparado el segundo objeto, B, con el primero, A, esta proporción debe ser reemplazada por sus recíprocos. Es natural asumir que en el juicio sintetizado también debe cambiar al recíproco. Por tanto, se requiere un método que permita desarrollar una regla para el juicio de grupo satisfaciendo esta propiedad recíproca.

Por ello, nos basamos en el trabajo de Saaty y Aczél [42] quienes demostraron que la media geométrica cumple con satisfacción la propiedad recíproca. En contraste, si se utiliza la media aritmética, esta propiedad no se mantiene. A manera de ejemplo, supongamos que con la media aritmética la media de dos juicios con valores de 7 y 9 resultaría en 8 , cuyo valor reciproco es $1 / 8$, pero en el otro lado de la matriz obtendríamos un valor de $(1 / 7+1 / 9) / 2=8 / 63$ lo que difiere del valor esperado de $1 / 8$ para mantener la matriz recíproca. Por otra parte, utilizando la media geométrica, la síntesis de estos dos juicios seria $\sqrt{7 \cdot 9}=3 \sqrt{7}$, y en al otro lado de la matriz el inverso estaría dado por $\sqrt{1 / 7 \cdot 1 / 9}=\sqrt{7 / 21}$, que es el mismo valor del reciproco esperado $1 / \sqrt{7 \cdot 9}=\sqrt{7 / 21}$.

Finalmente, calculamos un nuevo vector de prioridad consensuado obtenido para cada criterio, y se conforma una matriz de prioridad, la cual se multiplica con el vector de prioridad obtenido. El resultado final, será un vector denotado como vector de prioridad de las alternativas, el cual constituye la solución del problema.

\section{CASO DE APLICACIÓN}

Para validar lo desarrollado con anterioridad, se presenta un caso de estudio que consistió en seleccionar una técnica de modelamiento para el diseño de un modelo de inventario administrado por el proveedor (VMI) para una compañía retail colombiana. Esta elección estaba a cargo del equipo investigador, sin embargo, se propuso la consulta de un conjunto de expertos en el tema.

De manera inicial se contó con 19 expertos, su correcta selección fue crucial para reducir el error y la incertidumbre en la elección de la técnica. Por tanto, los expertos consultados contaban con campos de investigación relacionados con los objetivos del proyecto, la información se resume en la Tabla 4.

Tabla 4. Caracterización de expertos.

\begin{tabular}{|l|c|}
\hline \multicolumn{1}{|c|}{ Campo de Investigación } & $\begin{array}{c}\text { \% de } \\
\text { Expertos }\end{array}$ \\
\hline Sistemas productivos y logísticos. & $15,79 \%$ \\
\hline $\begin{array}{l}\text { Sistemas productivos y logísticos e Inves- } \\
\text { tigación de operaciones. }\end{array}$ & $57,89 \%$ \\
\hline Investigación de operaciones. & $21,05 \%$ \\
\hline Simulación. & $5,26 \%$ \\
\hline
\end{tabular}

Del mismo modo, contar con expertos con un alto nivel profesional, como se ve en la Figura 2, y experiencia, fue otra perspectiva a considerar. Por ello, se le pidió a cada uno valorar el grado de influencia para diferentes fuentes, como el conocimiento teórico, la experiencia práctica, su intuición sobre el tema o investigaciones llevadas a cabo, cuestionando el grado (alto, medio, bajo) en que han aportado a su conocimiento y juicio subjetivo sobre el tema.

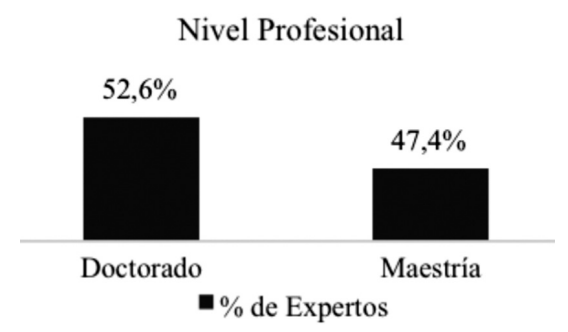

Figura 2. Estructura Jerárquica. 
En la selección de alternativas, se consideraron aquellas técnicas de modelamiento de la literatura científica consultada. Los modelos matemáticos analíticos (MMT) por optimización clásica, la Programación No Lineal (PNL), y la Teoría de Juegos (TJUE), fueron las técnicas más utilizadas por los autores para diseñar un modelo VMI y por lo tanto, fueron las elegidas para el juicio. Esto se sustenta en el hecho de contar con un enfoque recomendado, y en el que al mismo tiempo, sea posible contribuir en aspectos que aún no se hayan analizado o que convengan ser explorados.

Similarmente, para la selección de criterios se partió de aquellos implicados con frecuencia en la literatura consultada, con los cuales se construyó un listado inicial. El listado final fue obtenido mediante un consenso entre los participantes del proyecto, el cual se logró definiendo las necesidades o requerimientos del modelo. Los criterios seleccionados fueron los siguientes:

- Capacidad de modelar sistemas complejos (CMSC).

- Posibilidad de brindar una variedad de soluciones (VSOL).

- Fácil aplicabilidad (FAPL).

- Flexibilidad (FLEX).

Una estructura jerárquica ajustada al problema es la que se presenta en la Figura 3.

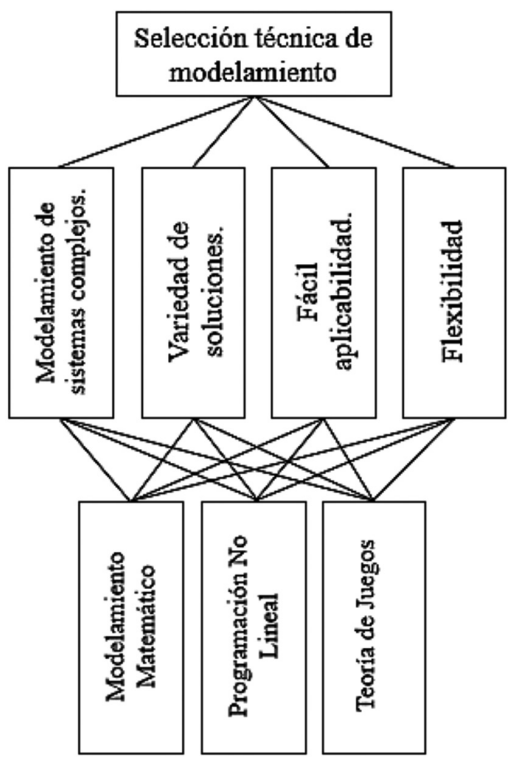

Figura 3. Estructura Jerárquica del caso.
$\mathrm{Al}$ aplicarse el algoritmo de consistencia, se evidenció que algunos expertos presentaban valores en la razón de consistencia mayores a 0,1 , el cual es el valor considerado no aceptable, como se mencionó anteriormente. Este hecho indicó que algunos de estos reflejaban contradicciones. Los resultados se resumen en la Tabla 5.

Para el cálculo de los valores de las matrices de valoraciones consensuadas, se procedió con la eliminación de aquellos resultados del conjunto de expertos que presentaron una o más matrices no consistentes. De manera posterior, se ejecutó la construcción de dichas matrices consensuadas, donde el valor de la comparación fue la media geométrica de la comparación de pares de los expertos que no presentaron inconsistencias, tal como se explicó con anterioridad. Los resultados para el conjunto de valoraciones se presentan de la Tabla 6 .

También, es importante aclarar que se le pidió a cada experto que marcara dentro de una encuesta de caracterización, el grado de conocimientos que este poseía acerca de los modelos de inventario administrado por el vendedor (Vendor Managed Inventory - VMI), valorándolo en una escala de 1 a 10 (considerando el número 1 como no poseer conocimiento, y el número 10 como el pleno conocimiento del tema tratado). Por otro lado, el sesgo por pertenecer a una misma filiación institucional supuso un problema en la emisión de juicios personales determinados por la orientación del perfil profesional, lineamientos investigativos, enfoque de los programas curriculares, entre otros factores. Por lo cual, se optó por la selección de expertos de diferentes instituciones de educación superior a lo largo del territorio nacional.

Luego de presentar las matrices de valoraciones consensuadas, de acuerdo con los fundamentos teóricos del proceso de jerarquía analítica (AHP), fue necesario normalizar las matrices, lo cual significa, dividir cada elemento de la matriz entre el valor total de su columna. Por otro lado, para el cálculo de los vectores de prioridad fue necesario promediar cada fila de la matriz normalizada. Los resultados de cada una de las matrices se presentan de la Tabla 7.

Ahora bien, se conforma una matriz de prioridad con cada vector de prioridad obtenido para los 
Tabla 5. Razón de consistencia de las valoraciones de los expertos.

\begin{tabular}{|c|c|c|c|c|c|c|c|c|c|c|}
\hline \multirow{3}{*}{ Experto } & & & \multicolumn{8}{|c|}{ Comparación entre alternativas } \\
\hline & \multicolumn{2}{|c|}{$\begin{array}{l}\text { Comparación } \\
\text { entre criterios }\end{array}$} & \multicolumn{2}{|c|}{ (CMSC) } & \multicolumn{2}{|c|}{ (FAPL) } & \multicolumn{2}{|c|}{ (FLEX) } & \multicolumn{2}{|c|}{ (VSOL) } \\
\hline & $\mathbf{C R}$ & Estado & CR & Estado & CR & Estado & $\mathbf{C R}$ & Estado & $\mathbf{C R}$ & Estado \\
\hline 1 & 0,056 & $\mathrm{C}$ & 0,000 & $\mathrm{C}$ & 0,010 & $\mathrm{C}$ & 0,063 & $\mathrm{C}$ & 0,039 & $\mathrm{C}$ \\
\hline 2 & 0,156 & $\mathrm{NC}$ & 0,000 & $\mathrm{C}$ & 0,098 & $\mathrm{C}$ & 0,993 & $\mathrm{NC}$ & 0,018 & $\mathrm{C}$ \\
\hline 3 & 0,016 & $\mathrm{C}$ & 0,017 & $\mathrm{C}$ & 0,008 & $\mathrm{C}$ & 0,016 & $\mathrm{C}$ & 0,042 & $\mathrm{C}$ \\
\hline 4 & 0,051 & $\mathrm{C}$ & 0,084 & $\mathrm{C}$ & 0,076 & $\mathrm{C}$ & 0,076 & $\mathrm{C}$ & 0,042 & $\mathrm{C}$ \\
\hline 5 & 0,046 & $\mathrm{C}$ & 0,084 & $\mathrm{C}$ & 0,042 & $\mathrm{C}$ & 0,032 & $\mathrm{C}$ & 0,073 & $\mathrm{C}$ \\
\hline 6 & 0,005 & $\mathrm{C}$ & 0,000 & $\mathrm{C}$ & 0,000 & $\mathrm{C}$ & 0,000 & $\mathrm{C}$ & 0,000 & $\mathrm{C}$ \\
\hline 7 & 0,459 & $\mathrm{NC}$ & 0,511 & $\mathrm{NC}$ & 0,365 & $\mathrm{NC}$ & 0,591 & $\mathrm{NC}$ & 0,012 & C \\
\hline 8 & 0,095 & $\mathrm{C}$ & 0,076 & $\mathrm{C}$ & 0,076 & $\mathrm{C}$ & 0,076 & $\mathrm{C}$ & 0,076 & $\mathrm{C}$ \\
\hline 9 & 0,037 & C & 0,007 & C & 0,017 & $\mathrm{C}$ & 0,016 & C & 0,000 & C \\
\hline 10 & 0,034 & $\mathrm{C}$ & 0,017 & $\mathrm{C}$ & 0,011 & $\mathrm{C}$ & 0,039 & $\mathrm{C}$ & 0,000 & $\mathrm{C}$ \\
\hline 11 & 0,099 & $\mathrm{C}$ & 0,033 & $\mathrm{C}$ & 0,005 & $\mathrm{C}$ & 0,048 & $\mathrm{C}$ & 0,087 & $\mathrm{C}$ \\
\hline 12 & 0,039 & $\mathrm{C}$ & 0,093 & $\mathrm{C}$ & 0,000 & $\mathrm{C}$ & 0,000 & $\mathrm{C}$ & 0,000 & $\mathrm{C}$ \\
\hline 13 & 0,073 & $\mathrm{C}$ & 0,077 & $\mathrm{C}$ & 0,077 & $\mathrm{C}$ & 0,077 & $\mathrm{C}$ & 0,077 & $\mathrm{C}$ \\
\hline 14 & 0,056 & $\mathrm{C}$ & 0,033 & $\mathrm{C}$ & 0,033 & $\mathrm{C}$ & 0,033 & $\mathrm{C}$ & 0,033 & $\mathrm{C}$ \\
\hline 15 & 0,299 & $\mathrm{NC}$ & 0,000 & $\mathrm{C}$ & 0,000 & $\mathrm{C}$ & 0,000 & $\mathrm{C}$ & 0,042 & $\mathrm{C}$ \\
\hline 16 & 0,000 & $\mathrm{C}$ & 0,432 & $\mathrm{NC}$ & 0,432 & $\mathrm{NC}$ & 0,432 & $\mathrm{NC}$ & 0,432 & $\mathrm{NC}$ \\
\hline 17 & 0,079 & $\mathrm{C}$ & 0,042 & $\mathrm{C}$ & 0,034 & $\mathrm{C}$ & 0,095 & $\mathrm{C}$ & 0,000 & $\mathrm{C}$ \\
\hline 18 & 0,000 & $\mathrm{C}$ & 0,000 & $\mathrm{C}$ & 0,000 & $\mathrm{C}$ & 0,000 & $\mathrm{C}$ & 0,000 & $\mathrm{C}$ \\
\hline 19 & 0,357 & $\mathrm{NC}$ & 0,462 & $\mathrm{NC}$ & 0,166 & $\mathrm{NC}$ & 0,055 & $\mathrm{C}$ & 0,468 & $\mathrm{NC}$ \\
\hline
\end{tabular}

Convenciones: $\mathrm{CC}=$ Razón de consistencia. Estados: $\mathrm{C}$, matriz consistente, $\mathrm{NC}$, matriz no consistente.

Tabla 6. Matrices de valoraciones consensuadas.

\begin{tabular}{|c|c|c|c|c|c|c|c|c|c|c|}
\hline \multicolumn{4}{|c|}{ Convenciones } & \multicolumn{7}{|c|}{$\begin{array}{l}\text { Matriz de valoraciones consensuadas: } \\
\text { Comparación de criterios. }\end{array}$} \\
\hline $\begin{array}{l}\text { CMSC } \\
\text { FAPL }\end{array}$ & \multicolumn{3}{|c|}{ Cap. de modelar sistemas complejos. } & & CMSC & \multicolumn{2}{|c|}{ FAPL } & \multicolumn{2}{|c|}{ FLEX } & VSOL \\
\hline FLEX & \multicolumn{3}{|c|}{ Flexibilidad. } & CMSC & 1 & \multicolumn{2}{|c|}{1,7573} & \multicolumn{2}{|c|}{2,2089} & 2,6031 \\
\hline VSOL & \multicolumn{3}{|c|}{ Variedad de soluciones. } & FAPL & 0,5690 & \multicolumn{2}{|c|}{1} & \multicolumn{2}{|c|}{1,8337} & 1,979 \\
\hline MMAT & \multicolumn{3}{|c|}{ Modelo matemático analítico. } & FLEX & 0,4527 & \multicolumn{2}{|c|}{0,5453} & \multicolumn{2}{|c|}{1} & 1,357 \\
\hline TJUE & \multicolumn{3}{|c|}{ Teoría de juegos. } & VSOL & 0,3841 & \multirow{2}{*}{\multicolumn{2}{|c|}{0,5051}} & \multicolumn{2}{|c|}{0,7367} & 1 \\
\hline PNL & \multicolumn{3}{|c|}{ Programación no lineal. } & & & & & & & \\
\hline \multicolumn{11}{|c|}{ Matriz de valoraciones consensuadas para cada uno de los criterios } \\
\hline \multicolumn{4}{|c|}{ Capacidad para modelar sistemas complejos. } & \multicolumn{7}{|c|}{ Fácil aplicabilidad. } \\
\hline & MMAT & TJUE & PNL & & \multicolumn{2}{|c|}{ MMAT } & \multicolumn{2}{|c|}{ TJUE } & \multicolumn{2}{|r|}{ PNL } \\
\hline MMAT & 1 & 2,3137274 & 1,23251 & MMAT & \multicolumn{2}{|l|}{1} & \multicolumn{2}{|c|}{1,7542252} & \multicolumn{2}{|c|}{1,652672} \\
\hline TJUE & 0,432203 & 1 & 0,709744 & TJUE & \multicolumn{2}{|c|}{0,570052} & \multicolumn{2}{|c|}{1} & \multicolumn{2}{|c|}{1,280887} \\
\hline PNL & 0,811352 & 1,4089588 & 1 & PNL & 0,6050 & & 0,78 & 7092 & 1 & \\
\hline \multicolumn{4}{|c|}{ Flexibilidad. } & \multicolumn{7}{|c|}{ Variedad de soluciones. } \\
\hline & MMAT & TJUE & PNL & & MMA & & & & & PNL \\
\hline MMAT & 1 & 2,7234164 & 3,135827 & MMAT & 1 & & 2,59 & 3451 & & 169068 \\
\hline TJUE & 0,367186 & 1 & 1,190065 & TJUE & 0,3860 & & 1 & & & 995084 \\
\hline PNL & 0,318895 & 0,8402899 & 1 & PNL & 0,4610 & & 1,00 & 9402 & 1 & \\
\hline
\end{tabular}


Tabla 7. Matrices normalizadas y vector de prioridad.

\begin{tabular}{|c|c|c|c|c|c|c|c|c|c|c|}
\hline \multicolumn{11}{|c|}{ Matriz normalizada y vector de prioridad para la comparación de criterios } \\
\hline & \multicolumn{2}{|r|}{ CMSC } & \multicolumn{2}{|c|}{ FAPL } & \multicolumn{2}{|c|}{ FLEX } & VSOL & \multicolumn{3}{|c|}{ Vector de prioridad } \\
\hline \multicolumn{2}{|c|}{ CMSC } & 0,42 & \multicolumn{2}{|c|}{0,46} & \multicolumn{2}{|c|}{0,38} & 0,38 & \multicolumn{3}{|c|}{0,409} \\
\hline \multicolumn{2}{|c|}{ FAPL } & 0,24 & \multicolumn{2}{|c|}{0,26} & \multicolumn{2}{|c|}{0,32} & 0,29 & \multicolumn{3}{|c|}{0,275} \\
\hline \multicolumn{2}{|c|}{ FLEX } & 0,19 & \multicolumn{2}{|c|}{0,14} & \multicolumn{2}{|c|}{0,17} & 0,20 & \multicolumn{3}{|c|}{0,175} \\
\hline \multicolumn{2}{|c|}{ VSOL } & 0,16 & \multicolumn{2}{|c|}{0,13} & \multicolumn{2}{|c|}{0,13} & 0,14 & \multicolumn{3}{|c|}{0,141} \\
\hline \multicolumn{2}{|c|}{ Total } & 1 & \multicolumn{2}{|c|}{1} & \multicolumn{2}{|c|}{1} & 1 & \multicolumn{3}{|c|}{1} \\
\hline \multicolumn{11}{|c|}{ Matriz normalizada y vector de prioridad para cada criterio } \\
\hline \multicolumn{6}{|c|}{ Capacidad para modelar sistemas complejos. } & \multicolumn{5}{|c|}{ Fácil aplicabilidad. } \\
\hline & MMAT & TJUE & PNL & \multicolumn{2}{|c|}{$\begin{array}{l}\text { Vector de } \\
\text { prioridad }\end{array}$} & & MMAT & TJUE & PNL & $\begin{array}{l}\text { Vector de } \\
\text { prioridad }\end{array}$ \\
\hline MMAT & 0,45 & 0,49 & 0,42 & \multicolumn{2}{|c|}{0,45} & MMAT & 0,46 & 0,50 & 0,42 & 0,46 \\
\hline TJUE & 0,19 & 0,21 & 0,24 & & & TJUE & 0,26 & 0,28 & 0,33 & 0,29 \\
\hline PNL & 0,36 & 0,30 & 0,34 & & & PNL & 0,28 & 0,22 & 0,25 & 0,25 \\
\hline Total & 1 & 1 & 1 & 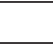 & & Total & 1 & 1 & 1 & 1 \\
\hline & & Flexibilic & & & & & Varie & ad de sol & aciones. & \\
\hline & MMAT & TJUE & PNL & & & & MMAT & TJUE & PNL & $\begin{array}{l}\text { Vector de } \\
\text { prioridad }\end{array}$ \\
\hline MMAT & 0,59 & 0,60 & 0,59 & & & MMAT & 0,54 & 0,56 & 0,52 & 0,54 \\
\hline TJUE & 0,22 & 0,22 & 0,22 & & & TJUE & 0,21 & 0,22 & 0,24 & 0,22 \\
\hline PNL & 0,19 & 0,18 & 0,19 & & & PNL & 0,25 & 0,22 & 0,24 & 0,24 \\
\hline Total & 1 & 1 & 1 & & & Total & 1 & 1 & 1 & 1 \\
\hline
\end{tabular}

criterios, la cual se multiplica con el vector de prioridad obtenido al realizar la comparación entre los criterios. El resultado, denominado vector de prioridad de las alternativas, constituirá la solución del juicio de expertos, al presentar cada una de las alternativas y un porcentaje de preferencia para cada una de ellas. La Tabla 8 contiene los resultados.

Por otro lado, el análisis de las valoraciones consensuadas no reflejó inconsistencias en la comparación de pares, es decir, las evaluaciones fueron consistentes y no existió contradicción en alguna en ellas. Esto se ilustra en la Tabla 9.

De acuerdo a las Tablas 8 y 9, la técnica que mejor se ajusta a los requerimientos proyectados, incluyendo los juicios de todos los expertos y presentando consistencia en sus valoraciones, fue el Modelo Matemático Analítico (MMAT) con un porcentaje de preferencia del $49,1 \%$, superior a la Teoría de Juegos (TJUE) y la Programación No lineal (PNL) con $23,8 \%$ y $27,1 \%$ respectivamente. Entonces, se puede inferir que el proceso ejecutado de juicio de
Tabla 8. Vector de prioridad de las alternativas.

\begin{tabular}{|l|l|}
\hline \multicolumn{2}{|c|}{ Ranking } \\
\hline Modelo matemático analítico & 0,491 \\
\hline Teoría de juegos & 0,238 \\
\hline Programación no lineal & 0,271 \\
\hline Total & 1 \\
\hline
\end{tabular}

Tabla 9. CR en valoraciones consensuadas.

\begin{tabular}{|l|c|c|}
\hline \multicolumn{1}{|c|}{ Matriz } & CR & Estado \\
\hline Comparación de criterios & 0,0072 & $\mathrm{C}$ \\
\hline $\begin{array}{l}\text { Capacidad para modelar sistemas } \\
\text { complejos }\end{array}$ & 0,0075 & $\mathrm{C}$ \\
\hline Fácil aplicabilidad & 0,0085 & $\mathrm{C}$ \\
\hline Flexibilidad & 0,0001 & $\mathrm{C}$ \\
\hline Variedad de soluciones & 0,0030 & $\mathrm{C}$ \\
\hline
\end{tabular}

expertos fue correctamente llevado a cabo para la selección de una técnica apropiada que cumpliera con un conjunto de criterios y alternativas distinguidas. 


\section{CONCLUSIONES}

Con respecto al caso de aplicación, podemos destacar que la aplicación de un juicio de expertos enfocado en un Proceso analítico jerárquico (AHP) constituyó un elemento de gran utilidad y de soporte en la elección del modelo matemático analítico como la técnica ideal para el diseño de un modelo de inventario administrado por el proveedor (VMI) que cumpliera con todos los criterios y elementos establecidos. A su vez, se logró un estudio analítico que reconoció todo un conjunto de juicios individuales de forma integral, sin caer en sesgos atribuidos a sofismas, juicios propios, u opiniones informales de los autores del proyecto.

Ahora bien, existe una serie de limitaciones en estudios de este tipo, ya que no se debe olvidar que la metodología desarrollada e implementada debe trabajarse como un complemento de la selección, dado que es conveniente tener muy claro las distintas alternativas y criterios para la toma de decisiones. Por tanto, se recomienda tener fuentes de información propicias en las fases de diagnóstico de criterios $\mathrm{y}$ alternativas, permitiendo tener una perspectiva correcta de los elementos ideales para cualquier estudio. A su vez, al reconocer que algunas técnicas de selección pueden tener una validez limitada y excluyente, el recurrir a una técnica como el Proceso analítico jerárquico (AHP) es quizás la estrategia más adecuada para obtener opiniones agregadas de la mejor calidad.

La relevancia del estudio gira en torno al aporte de un caso de aplicación y metodología que ayude a consolidar procesos de toma de decisiones estratégicas, que impacten y repercutan positivamente en cualquier caso de estudio o problema. Las organizaciones en general son sistemas abiertos que están en la constante necesidad de la toma de decisiones. Por tanto, con una estructura metodológica que soporte estos procesos, es posible obtener recomendaciones y resultados más satisfactorios, atribuidos a reducir el tiempo y esfuerzo, facilitar la descripción de cualquier problema de selección al dividirlo en criterios de decisión manejables, y sobre todo, la habilidad para que los tomadores de decisiones lleguen a un consenso con la documentación y justificación adecuada.

Finalmente, es posible identificar líneas de investigación para futuros estudios. El enfoque teórico y conceptual presentado para sintetizar los juicios a través de la media geométrica no cuenta con muchos casos de implementación en la literatura, por tanto, sería importante estudiar cómo se desarrollan en diversas situaciones problemas los distintos elementos identificados en el Proceso de jerarquía analítica (AHP). De forma complementaria, las diversas extensiones al AHP implementadas y propuestas en los últimos años (AHP-TOPSIS, fuzzy AHP, etc.), pueden ser aplicadas a estos problemas o al caso de aplicación desarrollado, para posteriormente comparar sus resultados y obtener inferencias relevantes.

\section{AGRADECIMIENTOS}

Los autores agradecen al conjunto de expertos quienes colaboraron para el desarrollo del caso de aplicación presentado.

\section{REFERENCIAS}

[1] M.A. Meyer and J.B. Brooker. "Eliciting and Analyzing Expert Judgment: A Practical Guide". ASA-SIAM Series. 1st ed. pp. 3-4. Estados Unidos. ISBN: 0898714745. 2001.

[2] S. Parthasarathy and S. Sharma. "Determining ERP customization choices using nominal group technique and analytical hierarchy process". Computers in Industry. Vol. $65 \mathrm{~N}^{\circ} 6$, pp. 1009-1017. Agosto 2014. ISSN: 01663615. DOI:10.1016/j.compind.2014.03.003.

[3] O.U. Akaa, A. Abu, M. Spearpoint and S. Giovinazzi. "A group-AHP decision analysis for the selection of applied fire protection to steel structures". Fire Safety Journal. Vol. 86, pp. 95-105. Noviembre 2016. ISSN: 03797112. DOI: 10.1016/j.firesaf.2016.10.005.

[4] B. R. Meesariganda and A. Ishizaka. "Mapping verbal AHP scale to numerical scale for cloud computing strategy selection". Applied Soft Computing. Vol. 53, pp. 111-118. Abril 2017. ISSN: 1568-4946. DOI: 10.1016/j. asoc.2016.12.040.

[5] Ö.F. Gürcan, İ. Yazıcı, Ö.F. Beyca, Ç.Y. Arslan and F. Eldemir. "Third Party Logistics (3PL) Provider Selection with AHP Application, Procedia". Social and Behavioral Sciences. Vol. 235, pp. 226-234. Noviembre 2016. ISSN: 1877-0428. DOI: 10.1016/j.sbspro.2016.11.018. 
[6] J.G. Vidal, M. Ramos, J.E. Azevedo and P.C. Cabral. "An AHP-based framework for logistics operations in distribution centres". International Journal of Production Economics. Vol. 187, pp. 246-259. Mayo 2017. ISSN: 0925-5273. DOI: 10.1016/j.ijpe.2017.03.001.

[7] K.K. Kim, C.A. O'Bryan, P.G. Crandall, S.C. Ricke and J.A. Neal. "Identifying baseline food safety training practices for retail delis using the Delphi expert consensus method". Food Control. Vol. $32 \mathrm{~N}^{\mathrm{o}}$ 1, pp. 55-62. Julio 2013. ISSN: 0956-7135. DOI: 10.1016/j. foodcont.2012.10.009.

[8] M. Kim, Y.C. Jang and S. Lee. "Application of Delphi-AHP methods to select the priorities of WEEE for recycling in a waste management decision-making tool". Journal of Environmental Management. Vol. 128, pp. 941-948. Octubre 2013. ISSN: 03014797. DOI: 10.1016/j.jenvman.2013.06.049.

[9] S. Di, J.D. Castillo and L. Lamelza. "Real Time Spatial Delphi: Fast convergence of experts' opinions on the territory". Technological Forecasting and Social Change. Vol. 115, pp. 143-154. Febrero 2017. ISSN: 00401625. DOI: $10.1016 / j$. techfore.2016.09.029.

[10] V. Mani, R. Agrawal and V. Sharma. "Supplier selection using social sustainability: AHP based approach in India". International Strategic Management Review. Vol. $2 \mathrm{~N}^{\circ} 2$, pp. 98-112. Diciembre 2014. ISSN: 23067748. DOI: 10.1016/j.ism.2014.10.003.

[11] I. Așchilean, G. Badea, I. Giurca, G.S. Naghiu and F.G Iloaie. "Choosing the Optimal Technology to Rehabilitate the Pipes in Water Distribution Systems Using the AHP Method". Energy Procedia. Vol. 112, pp. 19-26. Marzo 2017. ISSN: 1876-6102. DOI: 10.1016/j.egypro.2017.03.1109.

[12] B. Zhu and Z. Xu. "Analytic hierarchy processhesitant group decision making". European Journal of Operational Research. Vol. 239 $\mathrm{N}^{\mathrm{o}} 3$, pp. 794-801. Diciembre 2014. ISSN: 0377-2217. DOI: 10.1016/j.ejor.2014.06.019.

[13] R.P. Kusumawardani and M. Agintiara. "Application of Fuzzy AHP-TOPSIS Method for Decision Making in Human Resource Manager Selection Process". Procedia Computer Science. Vol. 72, pp. 638-646. Diciembre 2015. ISSN: 1877-0509. DOI: 10.1016/j.procs.2015.12.173.
[14] S. Kubler, J. Robert, W. Derigent, A. Voisin and Y.L. Traon. "A state-of the-art survey \& testbed of fuzzy AHP (FAHP) applications". Expert Systems with Applications. Vol. 65, pp 398-422. Diciembre 2016. ISSN: 09574174.DOI:10.1016/j.eswa.2016.08.064.

[15] C. Sekhar, M. Patwardhan and V. Vyas. "A Delphi-AHP-TOPSIS Based Framework for the Prioritization of Intellectual Capital Indicators: A SMEs Perspective". Procedia - Social and Behavioral Sciences. Vol. 189, pp. 275-284. Mayo 2015. ISSN: 1877-0428. DOI: 10.1016/j.sbspro.2015.03.223.

[16] E.R. Jalao, T. Wu and D. Shunk. "A stochastic AHP decision making methodology for imprecise preferences". Information Sciences. Vol. 270, pp. 192-203. Junio 2014. ISSN: 0020-0255. DOI: 10.1016/j.ins.2014.02.077.

[17] P. Ren, Z. Xu and H. Liao. "Intuitionistic multiplicative analytic hierarchy process in group decision making". Computers \& Industrial Engineering. Vol. 101, pp. 513524. Noviembre 2016. ISSN: 0360-8352. DOI: 10.1016/j.cie.2016.09.025.

[18] E.C. Özcan, S. Ünlüsoy and T. Eren. "A combined goal programming - AHP approach supported with TOPSIS for maintenance strategy selection in hydroelectric power plants". Renewable and Sustainable Energy Reviews. Vol. 78, pp. 1410-1423. Octubre 2017. ISSN: 1364-0321. DOI: 10.1016/j. rser.2017.04.039.

[19] Y. Bouzarour-Amokrane, A. Tchangani and F. Peres. "A bipolar consensus approach for group decision making problems". Expert Systems with Applications. Vol. $42 \mathrm{~N}^{\mathrm{o}} 3$, pp. 1759-1772. Febrero 2015. ISSN: 09574174. DOI: 10.1016/j.eswa.2014.09.061.

[20] J.V. Meijering, J.K. Kampen and H. Tobi. "Quantifying the development of agreement among experts in Delphi studies". Technological Forecasting and Social Change. Vol. $80 \mathrm{~N}^{\circ}$ 8, pp. 1607-1614. Octubre 2013. ISSN: 0040-1625. DOI: 10.1016/j. techfore.2013.01.003.

[21] N. Zhang, Z. Gong and F. Chiclana. "Minimum cost consensus models based on random opinions". Expert Systems with Applications. Vol. 89, pp. 149-159. Diciembre 2017. ISSN: 0957-4174. DOI: 10.1016/j. eswa.2017.07.035. 
[22] Z. Zhang and C. Wu. "A decision support model for group decision making with hesitant multiplicative preference relations". Information Sciences. Vol. 282, pp. 136-166. Octubre 2014. ISSN: 0020-0255. DOI: 10.1016/j.ins.2014.05.057.

[23] Y. Jiang, Z. Xu and X. Yu. "Compatibility measures and consensus models for group decision making with intuitionistic multiplicative preference relations". Applied Soft Computing. Vol. $13 \mathrm{~N}^{\circ}$ 4, pp. 20752086. Abril 2013. ISSN: 1568-4946. DOI: 10.1016/j.asoc.2012.11.007.

[24] W. Ho. "Integrated analytic hierarchy process and its applications - A literature review". European Journal of Operational Research. Vol. 186, Issue 1, pp. 211-228. Abril 2008. ISSN: 0377-2217. DOI: 10.1016/j. ejor.2007.01.004.

[25] A. Ishizaka and A. Labib. "Review of the main developments in the analytic hierarchy process". Expert Systems with Applications. Vol. $38 \mathrm{~N}^{\circ} 11$, pp. 14336-14345. Octubre 2011. ISSN: 0957-4174. DOI: 10.1016/j. eswa.2011.04.143.

[26] R. Russo and R. Camanho. "Criteria in AHP: A Systematic Review of Literature". Procedia Computer Science. Vol. 55, pp. 1123-1132. Julio 2015. ISSN: 1877-0509. DOI: 10.1016/j. procs.2015.07.081.

[27] M. Marttunen, J. Lienert and V. Belton. "Structuring problems for Multi-Criteria Decision Analysis in practice: A literature review of method combinations". European Journal of Operational Research. Vol. 263 $\mathrm{N}^{\circ}$ 1, pp. 1-17. Noviembre 2017. ISSN: 0377-2217. DOI: 10.1016/j.ejor.2017.04.041.

[28] M.I. De Arquer. "Fiabilidad humana: métodos de cuantificación, juicio de expertos". Notas Técnicas de Prevención. NTP. Centro Nacional de Condiciones de Trabajo. pp. 1-5. Madrid, Spain. 1996.

[29] F. Petropoulos, N. Kourentzes, K. Nikolopoulos and E. Siemsen. "Judgmental selection of forecasting models". Journal of Operations Management. Vol. 60, pp. 223-234. Junio 2018. ISSN: 0272-6963. DOI: $10.1016 / \mathrm{j}$. jom.2018.05.005

[30] C. Werner, T. Bedford, R.M. Cooke, A.M. Hanea and O. Morales. "Expert judgement for dependence in probabilistic modelling:
A systematic literature review and future research directions". European Journal of Operational Research. Vol. $258 \mathrm{~N}^{\mathrm{o}} 3$, pp. 801-819. Mayo 2017. ISSN: 0377-2217. DOI: 10.1016/j.ejor.2016.10.018.

[31] Y. Corral. "Validity and reliability of the instruments to collect data". Revista Ciencias de la Educación. Vol. 19 No 33, pp. 229-247. Enero 2009. ISSN: 1316-5917.

[32] H.A. Linstone and M. Turoff. "The Delphi method: Techniques and Applications". Advanced book program: Addison-Wesley. pp. 3-12. Estados Unidos. ISBN: 0-20104294-0. 1975.

[33] D. Barriga, A. L. Miranda y A. Bertha. "Metodología de la investigación educativa: Aproximaciones para comprender sus estrategias". Validez de expertos: algunos aportes teóricos y un caso concreto. Ediciones Díaz de Santos. pp. 168-171. Tlaxcala, México. ISBN: 978-84-9969-698-0. 2015

[34] M.A. Meyer and J.M. Booker. "Eliciting and Analyzing Expert Judgment: A Practical Guide". ASA-SIAM Series on Statistics and Applied Probability. pp. 99-122. London, Inglaterra. ISBN: 0-89871-474-5. 2001

[35] F. Bolger and G. Wright. "Expertise and Decision Support". Springer Science \& Business Media. pp. 168-176. New York, Estados Unidos. ISBN: 0-306-43862-3. 2007.

[36] A. Oliva, M. Ríos, L. Antolín, Á. Parra, Á. Hernando y M.Á. Pertegal. "Más allá del déficit: Construyendo un modelo de desarrollo positivo adolescente". Infancia y aprendizaje. Vol. $33 \mathrm{~N}^{\circ} 2$, pp. 223-234. Enero 2014. ISSN: 0210-3702. DOI: $10.1174 / 021037010791114562$.

[37] N. Harvey and C.A. Holmes. "Nominal group technique: An effective method for obtaining group consensus". International Journal of Nursing Practice. Vol. $18 \mathrm{~N}^{\mathrm{o}} 2$, pp. 188-194. Marzo 2012. ISSN: 1322-7114. DOI: 10.1111/j.1440-172X.2012.02017.

[38] D. Carter, J. Moizer and S. Liu. "Using groups to support judgmental parameter estimation VISCONS: Eyeballing to capture a quantified group consensus". Expert Systems with Applications. Vol. $40 \mathrm{~N}^{\mathrm{o}}$ 2, pp. 715721. Febrero 2013. ISSN: 0957-4174. DOI: 10.1016/j.eswa.2012.08.015.

[39] T. Rosqvist. "On the use of expert judgement in the qualification of risk assessment". 
VTT Publications. 1st ed. pp. 14-18. Espoo, Finlandia. ISBN: 951-38-6243-7. 2003.

[40] T.L. Saaty and L.G. Vargas. "Models, Methods, Concepts \& Applications of the Analytic Hierarchy Process". Springer: International Series in Operations Research \& Management Science. 2nd ed. pp. 5-11. New York, Estados Unidos. ISBN: 978-14614-3596-9. 2012.
[41] H.A. Taha. "Operations Research: An Introduction". Pearson Education. 8th ed. pp. 490-492. New Jersey, Estados Unidos. ISBN: 0-13-188923-0. 2007.

[42] J. Aczél and T.L. Saaty. "Procedures for synthesizing ratio judgements". Journal of Mathematical Psychology. Vol. $27 \mathrm{~N}^{\mathrm{o}} 1$, pp. 93-102. Marzo 1983. ISSN: 0022-2496. DOI:10.1016/0022-2496(83)90028-7. 